\title{
PLANE WAVES IN LINEAR VISCOELASTIC MATERIALS*
}

\author{
$\mathrm{BY}$
}

M. A. HAYES (University of East Anglia, Norwich)

AND

\section{R. S. RIVLIN (Lehigh University)}

1. Introduction. In this paper we discuss the propagation of plane sinusoidal waves in linear viscoelastic materials, both anisotropic and isotropic. Unlike the usual discussions (see, for example, [1]), we do not here assume that the planes of constant amplitude and constant phase are parallel. We do, however, assume that the imaginary parts of the complex moduli are small compared with their real parts and that correspondingly the magnitude of the imaginary part of the slowness vector is small compared with that of the real part. This implies that the attenuation of the wave is small in distances of travel of the order of a wavelength.

For a general anisotropic material it is found that, provided the imaginary parts of the complex moduli are sufficiently small compared with their real parts, for any specified directions of the normals to the planes of constant phase and constant amplitude, except for those satisfying a certain relation which depends on the real part of the complex modulus tensor, three waves can be propagated. Each of these is slightly elliptically polarized, the major axis of the ellipse being large compared with its minor axis.

In the case of an isotropic material, one of these waves is nearly longitudinal and the others are nearly transverse waves. The nearly longitudinal wave is slightly elliptically polarized with its major axis in the longitudinal direction and its minor axis coplanar with the normals to the planes of constant amplitude and constant phase. The nearly transverse waves are also slightly elliptically polarized with their major axes arbitrarily oriented in the planes of constant phase and their minor axes in the direction of propagation. For these waves, both the real and imaginary parts of the complex slowness vector are independent of the orientation of the direction, in the transverse plane, of the major axis of the ellipse. Consequently, waves may also be propagated which result from the superposition of two such nearly transverse waves, for which the displacements in the transverse plane have different directions of polarization and different phases.

In an isotropic viscoelastic material, for which the imaginary parts of the Lamé constants are sufficiently small compared with their real parts, elliptically-polarized nearly longitudinal waves and elliptically-polarized nearly transverse waves of the type described can be propagated for any inclination, other than a right angle, of the normals to the planes of constant phase and constant amplitude. On the other hand, in an iso-

\footnotetext{
* Received January 24, 1973. This work was carried out with the support of a grant (GP-30904XI) from the National Science Foundation to Lehigh University.
} 
tropic elastic material, plane waves can only be propagated if the planes of constant phase are either parallel or perpendicular to planes of constant amplitude.

Lockett [2] has considered the propagation, in an isotropic linear viscoelastic material, of plane sinusoidal waves for which the planes of constant amplitude are not necessarily parallel to the planes of constant phase and has discussed the reflection-refraction problem for such waves. In his discussion he draws attention to the elliptical character of the polarization of the waves when the planes of constant phase and constant amplitude are not parallel. Lockett has not, however, made the assumptions which we have made here that the imaginary parts of the Lamé constants are small compared with the real parts and consequently his results are, in some respects, less explicit than ours. Also, he restricts his analysis to the case when the polarization is entirely in the plane formed by the normals to the planes of constant phase and constant amplitude.

2. Anisotropic materials. We consider a linear viscoelastic material undergoing a deformation for which the components of the displacement vector $\mathbf{u}(\tau)$ at time $\tau$, in a rectangular cartesian coordinate system $x$, are $u_{i}(\tau)$. It is assumed that the components $\sigma_{i i}$ of the stress tensor $\boldsymbol{\delta}$, at time $t$, are given by

$$
\sigma_{i i}=C_{i j k l} e_{k l}(t)+\int_{-\infty}^{t} f_{i j k l}(t-\tau) e_{k l}(\tau) d \tau,
$$

where $e_{k l}(\tau)$ are the components of the infinitesimal strain tensor e $(\tau)$ at time $\tau$, given by

$$
e_{k l}(\tau)=\frac{1}{2}\left[\frac{\partial u_{k}(\tau)}{\partial x_{l}}+\frac{\partial u_{l}(\tau)}{\partial x_{k}}\right] .
$$

$C_{i j k l}$ and $f_{i j k l}$ are symmetric with respect to interchange of $i$ and $j$ and of $k$ and $l$.

We now suppose that the deformation corresponds to a damped plane sinusoidal wave of angular frequency $\omega$. Using the usual complex notation, we can write the complex displacement $u_{i}(\tau)$ in the form

$$
u_{i}(\tau)=U_{i} \exp \iota \omega\left(S_{k} x_{k}-\tau\right),
$$

where $U_{i}$ is a complex constant vector and $S_{k}$ are the components in the system $x$ of the complex slowness vector $\mathbf{S}$.

Introducing (2.3) and (2.2) into (2.1), we see that the complex stress is given by

$$
\sigma_{i i}=\Sigma_{i j} \exp \iota \omega\left(S_{k} x_{k}-t\right),
$$

where

$$
\Sigma_{i i}=\iota \omega c_{i j k l} S_{l} U_{k}
$$

and

$$
c_{i j k l}=c_{i j k l}(\omega)=\left[C_{i j k l}+\int_{-\infty}^{t} f_{i j k l}(t-\tau) \exp \iota \omega(t-\tau) d \tau\right] .
$$

$c_{i j k l}$ is the complex modulus tensor for the material.

In the absence of body forces, the stress $\sigma_{i i}$ must satisfy the equations of motion

$$
\partial \sigma_{i j} / \partial x_{i}=\rho\left(\partial^{2} u_{i} / \partial t^{2}\right),
$$


where $u_{i}=u_{i}(t)$ and $\rho$ denotes the mass density of the material. Introducing (2.3), (2.4) and (2.5) into this equation, we obtain

$$
\left(c_{i j k l} S_{i} S_{l}-\rho \delta_{i k}\right) U_{k}=0 .
$$

This has a non-trivial solution for $U_{k}$ if and only if

$$
\left|c_{i j k l} S_{i} S_{l}-\rho \delta_{i k}\right|=0 .
$$

We shall assume that the planes of constant phase and the planes of constant amplitude for the wave are not necessarily the same and that they are normal to the real vectors $\mathrm{m}$ and $\mathrm{n}$ respectively, which have components $m_{i}$ and $n_{i}$ respectively in the system $x$. We shall also assume that, for the wave considered, the imaginary part of $\mathbf{S}$ is small in magnitude compared with the real part and that the imaginary part of $c_{i j k l}$ is small compared with the real part. We may then write

$$
\begin{gathered}
\mathbf{S}=\mathbf{S}^{+}+\imath \epsilon \mathbf{S}^{-}=S^{+} \mathbf{m}+\imath \epsilon S^{-} \mathbf{n} \\
c_{i j k l}=c_{i j k l}{ }^{+}+\imath \epsilon c_{i j k l}{ }^{-},
\end{gathered}
$$

where $\epsilon$ is small and real. We assume that the elastic part $c_{i j k l}{ }^{+}$of the complex modulus satisfies the Onsager relations

$$
c_{i j k l}{ }^{+}=c_{k l i i}{ }^{+} .
$$

It follows from (2.8) that we may, without loss of generality, take the imaginary part of $\mathrm{U}$ to be $O(\epsilon)$. We may therefore write

$$
\mathbf{U}=\mathbf{U}^{+}+\iota \epsilon \mathbf{U}^{-} \text {. }
$$

Introducing (2.10) and (2.12) into (2.8), we obtain, by equating the coefficients of $\epsilon^{0}$ and $\epsilon$ separately to zero, the propagation conditions

$$
\begin{gathered}
\left\{\left(S^{+}\right)^{2} c_{i j k l}{ }^{+} m_{l} m_{i}-\rho \delta_{i k}\right\} U_{k}^{+}=0, \\
S^{+}\left\{S^{-} c_{i j k l}{ }^{+}\left(m_{l} n_{i}+m_{j} n_{l}\right)+S^{+} c_{i j k l}{ }^{-} m_{l} m_{j}\right\} U_{k}{ }^{+}=-\left\{\left(S^{+}\right)^{2} c_{i j k l}{ }^{+} m_{l} m_{j}-\rho \delta_{i k}\right\} U_{k}{ }^{-} .
\end{gathered}
$$

3. The energy flux vector. The energy flux vector $R_{i}(t)$ at time $t$ is defined as the rate at which energy leaves the material across an element of area normal to the $x_{i}$-axis, measured per unit area. It is given by

$$
R_{i}(t)=-\sigma_{i i}{ }^{+}(t) \dot{u}_{i}^{+}(t) .
$$

This fluctuates with time and accordingly we define a mean energy flux vector $\tilde{R}$ as the average of $\mathbf{R}(t)$ over a cycle. Thus

$$
\tilde{R}_{i}=\frac{\omega}{2 \pi} \int_{0}^{2 \pi / \omega} R_{i}(t) d t .
$$

Inserting (2.3) and (2.4) in (3.1), we find from (3.2) that

$$
\tilde{R}_{i}=\frac{1}{2} \omega^{2}\left(c_{i j k l} S_{l} U_{k} \bar{U}_{i}\right)^{+} \exp \left(-2 \epsilon \omega S_{p}{ }^{-} x_{p}\right),
$$

where the bar denotes the complex conjugate. By using (2.10), (2.11) and (2.12), Eq. (3.3) becomes 


$$
\tilde{R}_{i}=\frac{1}{2} \omega^{2} S^{+} c_{i j k l}{ }^{+} m_{l} U_{k}{ }^{+} U_{i}{ }^{+} \exp \left(-2 \epsilon \omega S_{p}{ }^{-} x_{p}\right),
$$

neglecting terms of first and higher orders in $\epsilon$ but bearing in mind that $x_{p}$ may be large. We note, using (2.11), that

$$
\begin{aligned}
\widetilde{R}_{i} n_{i} & =\frac{1}{2} \omega^{2} S^{+} c_{i j k l}{ }^{+} n_{i} m_{l}{U_{k}}^{+} U_{i}{ }^{+} \exp \left(-2 \epsilon \omega S_{p}{ }^{-} x_{p}\right) \\
& =\frac{1}{2} \omega^{2} S^{+} c_{i j k l}{ }^{+} m_{l} n_{j} U_{i}{ }^{+} U_{k}{ }^{+} \exp \left(-2 \epsilon \omega S_{p}{ }^{-} x_{p}\right) .
\end{aligned}
$$

The stress power $\mathscr{D}$ is given by

$$
D=\sigma_{i i}{ }^{+}\left(\partial \dot{u}_{i}{ }^{+} / \partial x_{i}\right)
$$

Thus if $\widetilde{D}$ denotes the amount of energy dissipated in a cycle, per unit volume, we have

$$
\tilde{D}=\int_{0}^{2 \pi / \omega} \sigma_{i i}{ }^{+} \frac{\partial \dot{u}_{i}^{+}}{\partial x_{i}} d t .
$$

Using (2.3) and (2.4) in (3.7), we find

$$
\widetilde{D}=-\pi \omega^{2}\left(c_{i j k l} S_{l} U_{k} \bar{U}_{i} \bar{S}_{i}\right)^{-} \exp \left(-2 \epsilon \omega S_{p}{ }^{-} x_{p}\right) .
$$

To the first order in $\epsilon$, we obtain, with (2.11),

$$
\tilde{\mathbb{D}}=-\epsilon \pi \omega^{2}\left(S^{+}\right)^{2} c_{i j k l}{ }^{-} m_{l} m_{i} U_{i}{ }^{+} U_{k}{ }^{+} \exp \left(-2 \epsilon \omega S_{p}{ }^{-} x_{p}\right) .
$$

4. The propagation conditions. We now consider the propagation conditions (2.13) in detail.

If the unit vector $\mathrm{m}$ is specified, $S^{+}$may be obtained from the secular equation

$$
\left|\left(S^{+}\right)^{2} c_{i j k l}{ }^{+} m_{l} m_{i}-\rho \delta_{i k}\right|=0,
$$

which is the condition that $(2.13)_{1}$ have a solution other than $U_{k}^{+}=0$. From (2.11) it follows that

$$
c_{i j k l}{ }^{+} m_{l} m_{i}=c_{k i i l}{ }^{+} m_{l} m_{i},
$$

and hence the three roots of $(4.1)$ for $\left(S^{+}\right)^{2}$ are all real. To each of these roots there corresponds a solution of $(2.13)_{1}$ for $U_{k}{ }^{+}$which is determined uniquely in direction but not in magnitude. These three directions are, of course, mutually perpendicular.

We now multiply $(2.13)_{2}$ by $U_{i}{ }^{+}$. Then using (2.11), (2.13) ${ }_{1}$ and (4.2), and assuming $S^{+} \neq 0$, we obtain

$$
2 S^{-} c_{i j k l}{ }^{+} m_{l} n_{j} U_{i}{ }^{+} U_{k}{ }^{+}=-S^{+} c_{i j k l}{ }^{-} m_{l} m_{i} U_{i}{ }^{+} U_{k}{ }^{+} .
$$

We can eliminate $U_{i}{ }^{+} U_{k}{ }^{+}$from Eq. (4.3) in the following manner. Let $\lambda_{i k}$ denote the cofactor of $\left(S^{+}\right)^{2} c_{i j k l} m_{l} m_{i}-\rho \delta_{i k}$ in $\operatorname{det}\left|\left(S^{+}\right)^{2} c_{i j k l} m_{l} m_{i}-\rho \delta_{i k}\right|$. Then, following Synge [2], we can express $\lambda_{i k}$ in the form

$$
\lambda_{i k}=\theta U_{i}^{+} U_{k}^{+},
$$

where $\theta$ is a constant. Accordingly (4.3) may be rewritten as

$$
2 S^{-} c_{i j k l}{ }^{+} m_{l} n_{j} \lambda_{i k}=-S^{+} c_{i j k l}{ }^{-} m_{l} m_{i} \lambda_{i k} .
$$

Eq. (4.3) determines $S^{-}$in terms of $S^{+}, \mathrm{m}$ and $\mathrm{n}$ provided that

$$
c_{i j k l}{ }^{+} m_{l} n_{i} U_{i}{ }^{+} U_{k}^{+} \neq 0 \text {. }
$$


Since $\mathbf{U}^{+}$satisfies $(2.13)_{1}$, it can be shown that the component of $\mathbf{U}^{-}$in the direction of $\mathrm{U}^{+}$is not determined by $(2.13)_{2}$. In order to do this, we write $\mathrm{U}^{-}$in the form

$$
\mathbf{U}^{-}=\alpha \mathbf{U}^{+}+\mathbf{P}
$$

where $\mathbf{P}$ is a real vector perpendicular to $\mathbf{U}^{+}$and lying in the plane of $\mathbf{U}^{-}$and $\mathbf{U}^{+}$, so that

$$
\text { P. } \mathbf{U}^{+}=0 \text {. }
$$

Introducing (4.7) into $(2.13)_{2}$ and using $(2.13)_{1}$, we obtain

$$
\begin{aligned}
S^{+}\left\{S^{-} c_{i j k l}{ }^{+}\left(m_{l} n_{j}+m_{j} n_{l}\right)+S^{+} c_{i j k l}{ }^{-} m_{l} m_{j}\right\} U_{k}{ }^{+} & \\
& =-\left\{\left(S^{+}\right)^{2} c_{i j k l}{ }^{+} m_{l} m_{i}-\rho \delta_{i k}\right\} P_{k} .
\end{aligned}
$$

Thus, Eq. $(2.13)_{2}$, which appears superficially to be an equation for the determination of $\mathbf{U}^{-}$if $\mathbf{U}^{+}, \mathbf{m}$ and $\mathbf{n}$ are known, in fact determines only the component of $\mathbf{U}^{-}$in the plane perpendicular to $\mathrm{U}^{+}$. This leaves the component of $\mathrm{U}^{-}$parallel to $\mathrm{U}^{+}$, and hence the phase of the component of $\mathrm{U}$ in this direction, undetermined.

Suppose now that we have chosen $\mathrm{m}$ and solved (4.1) for $\mathrm{S}^{+}$and $(2.13)_{1}$ for $\mathrm{U}^{+}$. Then there is a whole plane of directions $\mathbf{n}$ for which (4.6) is not satisfied, i.e. for which

$$
c_{i j k l}{ }^{+} m_{l} n_{i} U_{i}{ }^{+} U_{k}{ }^{+}=0 .
$$

In general, however, the corresponding wave

$$
u_{i}=\left(U_{i}^{+}+\iota \epsilon U_{i}^{-}\right) \exp \iota \omega\left(S^{+} m_{p} x_{p}-t\right) \exp \left(-\epsilon S^{-} n_{p} x_{p}\right)
$$

does not propagate in the material. For, if (4.10) holds, then, from (4.3),

$$
S^{+} c_{i j k l}{ }^{-} m_{l} m_{i} U_{i}{ }^{+} U_{k}{ }^{+}=0
$$

must be satisfied. Here $\mathrm{m}$ and $\mathrm{U}^{+}$are assumed known so that in general (4.12) cannot be satisfied unless $S^{+}=0$, in which case the wave does not progress.

From (3.5) it is seen that (4.10) may be written

$$
\tilde{R}_{i} n_{i}=0,
$$

so that the mean energy flux vector lies in the plane of constant amplitude, whilst by (3.9) Eq. (4.12) may be written

$$
\widetilde{D}=0 \text {, }
$$

so that no energy is dissipated. So, if for some choice of $\mathbf{m}$ and corresponding solutions $S^{+}$of (4.1) and $\mathrm{U}^{+}$of $(2.13)_{1} \mathrm{n}$ is chosen to satisfy (4.10) and if (4.12) holds, then the material behaves elastically for the corresponding wave.

Of course, if (4.10) holds, then (4.3) may not be used to determine $S^{-}$. In fact $S^{-}$ is arbitrary and must be determined from the boundary conditions. This arbitrariness in $S^{-}$leads to a corresponding arbitrariness in $P_{k}$, from (4.9).

The indeterminacy of $\mathrm{U}$ is, in fact, more apparent than real. We note, from (2.8), that for a specified $\mathbf{S}$ satisfying (2.9), $\mathbf{U}$ is determined apart from an arbitrary scalar multiplier. The apparent indeterminacy of the component $\alpha \mathrm{U}^{+}$of $\mathrm{U}^{-}$(see (4.7)) results from the approximation in which terms of order $\epsilon^{2}$ are neglected. To the order $\epsilon, \mathbf{P}$ is unaltered by small changes of phase in the component of $\mathrm{U}^{-}$parallel to $\mathrm{U}^{+}$, but it would be altered if terms of higher order in $\epsilon$ were retained. 
The fact that the component of $\mathrm{U}$ perpendicular to $\mathrm{U}^{+}$is not zero implies that the wave is slightly elliptically polarized, the major axis of the ellipse being substantially in the direction of $\mathbf{U}^{+}$.

For an elastic material $c_{i j k l}{ }^{-}=0$, and from (4.3) we obtain

$$
S^{-}=0 \text {, }
$$

or

$$
c_{i j k l}{ }^{+} m_{l} n_{j} U_{i}^{+} U_{k}{ }^{+}=0 .
$$

If $S^{-}=0$, we have, from (4.9), $P_{k}=0$, so that $\mathrm{U}^{-}$and $\mathrm{U}^{+}$are parallel. We note that in general Eq. (2.13) ${ }_{1}$ yields three possible directions for $\mathbf{U}^{+}$, corresponding to the three possible solutions of $(4.1)$ for $\left(S^{+}\right)^{2}$. These are the directions of the three mutuallyperpendicular eigenvectors of the symmetric matrix $\left\|\left(S^{+}\right)^{2} c_{i j k l}{ }^{+} m_{l} m_{i}-\rho \delta_{i k}\right\|$. The corresponding waves have, of course, their complex displacements linearly polarized in the directions of these eigenvectors. The arbitrariness in the choice of $\mathrm{U}^{-}$corresponds to an arbitrariness in the phase of the wave.

If (4.16) is applicable, then for each $\mathrm{m}$ and corresponding $\left(S^{+}\right)^{2}$ and $\mathrm{U}^{+}$this relation determines the directions of $\mathbf{n}$ for which waves can be propagated in an elastic material. $S^{-}$is arbitrary and must be determined from the boundary conditions.

5. Isotropic materials. If the material is isotropic, we can write the complex modulus tensor $c_{i j k l}$ in the form

$$
c_{i j k l}=\mu\left(\delta_{i k} \delta_{j l}+\delta_{j k} \delta_{i l}\right)+\lambda \delta_{i j} \delta_{k l},
$$

where $\mu$ and $\lambda$ are the complex Lamé constants for the material. We introduce the notation

$$
\mu=\mu^{+}+\iota \epsilon \mu^{-}, \quad \lambda=\lambda^{+}+\iota \epsilon \lambda^{-} .
$$

Eqs. (2.13) then become

$$
\begin{aligned}
&\left(S^{+}\right)^{2}\left[\left(\mu^{+}+\lambda^{+}\right)\left(\mathbf{U}^{+} \cdot \mathbf{m}\right) \mathbf{m}+\mu^{+} \mathbf{U}^{+}\right]-\rho \mathbf{U}^{+}=\mathbf{0}, \\
& S^{+} S^{-}\left[2 \mu^{+}(\mathbf{m} \cdot \mathbf{n}) \mathbf{U}^{+}\right.\left.+\left(\mu^{+}+\lambda^{+}\right)\left\{\left(\mathbf{U}^{+} \cdot \mathbf{m}\right) \mathbf{n}+\left(\mathbf{U}^{+} \cdot \mathbf{n}\right) \mathbf{m}\right\}\right] \\
&+\left(S^{+}\right)^{2}\left[\mu^{-} \mathbf{U}^{+}+\left(\mu^{-}+\lambda^{-}\right)\left(\mathbf{U}^{+} \cdot \mathbf{m}\right) \mathbf{m}\right] \\
&=\left[\rho-\mu^{+}\left(S^{+}\right)^{2}\right] \mathbf{U}^{-}-\left(\mu^{+}+\lambda^{+}\right)\left(S^{+}\right)^{2}\left(\mathbf{U}^{-} \cdot \mathbf{m}\right) \mathbf{m} .
\end{aligned}
$$

Also, Eq. (4.1) becomes

$$
\left|\left(S^{+}\right)^{2}\left[\left(\mu^{+}+\lambda^{+}\right) m_{i} m_{k}+\mu^{+} \delta_{i k}\right]-\rho \delta_{i k}\right|=0 .
$$

By using (3.9), the dissipation per cycle, per unit volume, is given by

$$
\tilde{D}=-\epsilon \pi \omega^{2}\left(S^{+}\right)^{2}\left\{\mu^{-} \mathbf{U}^{+} \cdot \mathbf{U}^{+}+\left(\lambda^{-}+\mu^{-}\right)\left(\mathbf{U}^{+} \cdot \mathbf{m}\right)^{2}\right\} \exp \left(-2 \epsilon \omega \mathbf{S}^{-} \cdot \mathbf{x}\right)
$$

and, from (3.4), the mean energy flux vector is

$$
\tilde{\mathbf{R}}=\frac{1}{2} \omega^{2} S^{+}\left\{\left(\lambda^{+}+\mu^{+}\right)\left(\mathbf{U}^{+} \cdot \mathbf{m}\right) \mathbf{U}^{+}+\mu^{+}\left(\mathbf{U}^{+} \cdot \mathbf{U}^{+}\right) \mathbf{m}\right\} \exp \left(-2 \epsilon \omega \mathbf{S}^{-} \cdot \mathbf{x}\right) .
$$

Eq. (5.4) yields two different solutions for $\left(S^{+}\right)^{2}$ and it is seen from (5.3) ${ }_{1}$ that these correspond to waves for which the vector $\mathrm{U}^{+}$is polarized transversely and longitudinally 
with respect to $\mathbf{m}$, the unit normal to the planes of constant phase. We shall discuss the two types of wave separately.

(i) Transverse waves. In this case, we have

$$
\mu^{+}\left(S^{+}\right)^{2}=\rho, \quad \mathrm{U}^{+} \cdot \mathrm{m}=0 .
$$

Eq. (5.3) $)_{2}$ then becomes

$S^{-}\left[2 \mu^{+}(\mathbf{m} \cdot \mathbf{n}) \mathbf{U}^{+}+\left(\mu^{+}+\lambda^{+}\right)\left(\mathbf{U}^{+} \cdot \mathbf{n}\right) \mathbf{m}\right]+S^{+} \mu^{-} \mathbf{U}^{+}=-S^{+}\left(\mu^{+}+\lambda^{+}\right)\left(\mathbf{U}^{-} \cdot \mathbf{m}\right) \mathbf{m}$,

and (5.5) and (5.6) become

$$
\begin{aligned}
& \tilde{\mathbb{D}}=-\epsilon \pi \omega^{2}\left(S^{+}\right)^{2} \mu^{-} \mathbf{U}^{+} \cdot \mathbf{U}^{+} \exp \left(-2 \epsilon \omega \mathbf{S}^{-} \cdot \mathbf{x}\right), \\
& \widetilde{R}=\frac{1}{2} \omega^{2} S^{+} \mu^{+}\left(\mathbf{U}^{+} \cdot \mathbf{U}^{+}\right) \mathbf{m} \exp \left(-2 \epsilon \omega \mathbf{S}^{-} \cdot \mathbf{x}\right) .
\end{aligned}
$$

Taking the inner product of $(5.8)$ with $\mathrm{U}^{+}$and using $(5.7)_{2}$, we obtain

$$
2 \mu^{+} S^{-}(\mathbf{m} \cdot \mathbf{n})=-\mu^{-} S^{+} .
$$

Taking the inner product of (5.8) with $\mathrm{m}$ and using $(5.7)_{2}$, we obtain

$$
S^{+} \mathbf{U}^{-} \cdot \mathrm{m}=-S^{-} \mathbf{U}^{+} \cdot \mathbf{n} \text {. }
$$

Thus, provided

$$
\mathbf{m} \cdot \mathbf{n} \neq 0,
$$

Eq. (5.10) determines $S^{-}$and Eq. (5.11) determines the component of $\mathrm{U}^{-}$parallel to $\mathrm{m}$, i.e. normal to the planes of constant phase. The component of $\mathrm{U}^{-}$perpendicular to $\mathrm{m}$ is, however, not determined by the equations.

If $\mathbf{m} \cdot \mathbf{n}=0$, so that the condition (5.12) is violated, then, bearing in mind that $S^{+} \neq 0$, i.e. the wave cannot have zero phase velocity, we must have $\mu^{-}=0$. This condition implies that the material is elastic with respect to shear vibrations. Conversely, if $\mu^{-}=0$, then $S^{-}=0$, unless $\mathrm{m} \cdot \mathbf{n}=0$ (i.e., the planes of constant phase and constant amplitude are perpendicular). The latter condition arises in the case of Love and Rayleigh waves. If $S^{-}=0$, the planes of constant phase are also planes of constant amplitude and the wave propagates without change of amplitude. Also, from (5.11) it follows that $\mathbf{U}^{-} \cdot \mathbf{m}=0$, i.e. the wave is entirely transverse. We therefore conclude that for an elastic material, the waves must either have their planes of constant phase parallel or perpendicular to planes of constant amplitude. In the latter case, i.e., if $\mu^{-}=0$ and $\mathbf{m} \cdot \mathbf{n}=0$, then Eq. (5.10) is identically satisfied and does not enable us to determine $S^{-}$. The ratio $S^{-} /\left(\mathbf{U}^{-} \cdot \mathrm{m}\right)$ is determined from (5.11). $S^{-}$and the component of $\mathbf{U}^{-}$in the direction of $\mathbf{U}^{+}$must be determined from the boundary conditions.

In the case when $\mathbf{m} \cdot \mathbf{n} \neq 0$ and $\mu^{-} \neq 0$, i.e. the material is not elastic, it follows from (5.10) that $S^{-} \neq 0$. Then, from (5.10) and (5.11) it follows that

$$
\mathrm{U}^{-} \cdot \mathbf{m}=\frac{1}{2} \frac{\mu^{-}}{\mu^{+}} \frac{\mathrm{U}^{+} \cdot \mathbf{n}}{\mathrm{m} \cdot \mathbf{n}}
$$

The waves will be elliptically polarized, the plane of polarization being, in general, inclined at a small angle to the planes of constant phase. It follows from (5.13) that if $\mathbf{m}=\mathbf{n}$, so that $\mathrm{U}^{+} \cdot \mathbf{n}=0$, then $\mathrm{U}^{-} \cdot \mathbf{m}=0$ and the plane of polarization is perpendicular to the direction of propagation $\mathrm{m}$. We note also that if $\mu^{-} \neq 0$, the planes of constant 
amplitude and constant phase may be inclined to each other at any angle except a right angle. However, as far as the present discussion is concerned, this is restricted by the consideration that $\mathbf{m} \cdot \mathbf{n}$ must not be so small that the condition that the magnitude of $\epsilon \mathbf{U}^{-}$be small compared with that of $\mathbf{U}^{+}$is violated.

(ii) Longitudinal waves. In this case we have

$$
\left(\lambda^{+}+2 \mu^{+}\right)\left(S^{+}\right)^{2}=\rho, \quad \mathrm{U}^{+}=\beta \mathrm{m},
$$

say, where $\beta$ is a real constant. Then $(5.3)_{2}$ becomes

$$
\begin{aligned}
\beta S^{+} S^{-}\left[2 \mu^{+}(\mathbf{m} \cdot \mathbf{n}) \mathbf{m}+\left(\lambda^{+}+\mu^{+}\right)\{\mathbf{n}+(\mathbf{m} \cdot \mathbf{n}) \mathbf{m}\}\right]+\beta\left(S^{+}\right)^{2}\left(\lambda^{-}+2 \mu^{-}\right) \mathbf{m} \\
=\left[\rho-\mu^{+}\left(S^{+}\right)^{2}\right] \mathbf{U}^{-}-\left(\lambda^{+}+\mu^{+}\right)\left(S^{+}\right)^{2}\left(\mathbf{U}^{-} \cdot \mathbf{m}\right) \mathbf{m},
\end{aligned}
$$

and (5.5) and (5.6) become

$$
\begin{aligned}
& \tilde{D}=-\epsilon \pi \beta^{2} \omega^{2}\left(S^{+}\right)^{2}\left(\lambda^{-}+2 \mu^{-}\right) \exp \left(-2 \epsilon \omega \mathbf{S}^{-} \cdot \mathbf{x}\right), \\
& \tilde{\mathscr{R}}=\frac{1}{2} \beta^{2} \omega^{2} S^{+}\left(\lambda^{+}+2 \mu^{+}\right) \mathrm{m} \exp \left(-2 \epsilon \omega \mathbf{S}^{-} \cdot \mathbf{x}\right) .
\end{aligned}
$$

Multiplying (5.15) throughout by $\mathrm{m}$, we obtain, with (5.14)

$$
2 S^{-}\left(\lambda^{+}+2 \mu^{+}\right) \mathbf{m} \cdot \mathbf{n}=-S^{+}\left(\lambda^{-}+2 \mu^{-}\right) .
$$

Using (5.14) ${ }_{1}$ and assuming $S^{+} \neq 0$, we can rewrite (5.15) as

$$
\begin{aligned}
\beta S^{-}\left\{\left(\lambda^{+}+3 \mu^{+}\right)(\mathbf{m} \cdot \mathbf{n}) \mathbf{m}+\left(\lambda^{+}+\mu^{+}\right) \mathbf{n}\right\}+\beta S^{+}\left(\lambda^{-}+2 \mu^{-}\right) \mathbf{m} \\
=S^{+}\left(\lambda^{+}+\mu^{+}\right)\left[\mathbf{U}^{-}-\left(\mathbf{U}^{-} \cdot \mathbf{m}\right) \mathbf{m}\right] .
\end{aligned}
$$

We note that $\mathbf{U}^{-}-\left(\mathbf{U}^{-} \cdot \mathbf{m}\right) \mathbf{m}$ is the component of $\mathbf{U}^{-}$perpendicular to $\mathbf{m}$, i.e. to $\mathbf{U}^{+}$. Thus, provided

$$
\mathbf{m} \cdot \mathbf{n} \neq 0,
$$

Eq. (5.17) determines $S^{-}$and Eq. (5.18) enables us to determine the component of $\mathbf{U}^{-}$ perpendicular to $\mathbf{m}$, its component parallel to $\mathbf{m}$ (and hence the phase of $\mathbf{U} \cdot \mathbf{m}$ ) remaining undetermined.

We note also from (5.18) that $\left[\mathrm{U}^{-}-\left(\mathbf{U}^{-} \cdot \mathbf{m}\right) \mathbf{m}\right]$ lies in the plane of $\mathbf{m}$ and $\mathbf{n}$ and accordingly the wave is, in general, elliptically polarized in the plane of $\mathbf{m}$ and $\mathbf{n}$. However, in the particular case when $\mathbf{n}=\mathbf{m}$, i.e., the planes of constant phase and constant amplitude are parallel, it follows from (5.17) and (5.18) that $\mathbf{U}^{-}-\left(\mathbf{U}^{-} \cdot \mathbf{m}\right) \mathbf{m}=0$, so that the wave is linearly polarized in the direction of $\mathrm{m}$.

If $\mathbf{m} \cdot \mathbf{n}=0$, so that the condition (5.19) is violated, it follows from (5.17) that $\lambda^{-}+2 \mu^{-}=0$, i.e., the material is elastic with respect to longitudinal waves. Conversely, if the material is elastic so that $\lambda^{-}+2 \mu^{-}=0$, then either $\mathbf{m} \cdot \mathbf{n}=0$, or $S^{-}=0$. In the former case, the planes of constant phase and constant amplitude are perpendicular and we note that (5.17) is identically satisfied. Accordingly, Eqs. (5.17) and (5.18) cannot be used to determine $S^{-}$and $\mathrm{U}^{-}$. These must then be determined from the boundary conditions. If $S^{-}=0$, it follows from $(5.18)$ that $\left[\mathrm{U}^{-}-\left(\mathrm{U}^{-} \cdot \mathbf{m}\right) \mathrm{m}\right]=0$, i.e., the wave is longitudinally polarized.

In the case when $\lambda^{-}+2 \mu^{-} \neq 0$, i.e., the material is not elastic, it follows from (5.17) that $S^{-} \neq 0$. Then, from (5.17) and (5.18), we obtain 


$$
\mathbf{U}^{-}-\left(\mathbf{U}^{-} \cdot \mathbf{m}\right) \mathbf{m}=\frac{\alpha\left(\lambda^{-}+2 \mu^{-}\right)}{2\left(\lambda^{+}+2 \mu^{+}\right) \mathbf{m} \cdot \mathbf{n}}[(\mathbf{m} \cdot \mathbf{n}) \mathbf{m}-\mathbf{n}] .
$$

Accordingly, as in the case of transverse waves, the planes of constant phase and constant amplitude may be inclined to each other at any angle except a right angle. However, as far as the present discussion is concerned, this is restricted by the consideration that $\mathbf{m} \cdot \mathbf{n}$ must not be so small that the condition that the magnitude of $\epsilon \mathrm{U}^{-}$be small compared with that of $\mathbf{U}^{+}$is violated.

\section{REFERENCES}

[1] D. R. Bland, The theory of linear viscoelasticity, Pergamon Press, Oxford (1960)

[2] J. L. Synge, Proc. Royal Irish Acad. 58A, 13 (1956)

[3] F. J. Lockett, J. Mech. Phys. Solids 10, 53 (1962) 